\title{
The Existence of Solution of Diffusion Equation with the General Conformable Derivative
}

\author{
Shanshan Li $\mathbb{D}^{1}{ }^{1}$ Shuqin Zhang, ${ }^{2}$ and Ruiying Liu ${ }^{3}$ \\ ${ }^{1}$ School of Mathematics and Information Sciences, Yantai University, 264005 Yantai, China \\ ${ }^{2}$ School of Science, China University of Mining and Technology, 100083 Beijing, China \\ ${ }^{3}$ Lang Yue Hui Foreign Language Middle School, 450001 Zhengzhou, China
}

Correspondence should be addressed to Shanshan Li; shanhuyuli@163.com

Received 22 March 2020; Accepted 22 April 2020; Published 11 May 2020

Academic Editor: Liguang Wang

Copyright (c) 2020 Shanshan Li et al. This is an open access article distributed under the Creative Commons Attribution License, which permits unrestricted use, distribution, and reproduction in any medium, provided the original work is properly cited.

In this paper, the general conformable fractional derivative is used in the classical diffusion equations, and the corresponding maximum principle is obtained. By the maximum principle, this paper proves the uniqueness of the solution and the continuous dependence on source function and initial-boundary conditions of the solution. Furthermore, by employing the variable separation method, this paper obtains some existence results and the asymptotic behavior of the classical solution.

\section{Introduction}

It has been several decades since the fractional calculus has attracted extensive attention in both pure and applied fields $[1-8]$. Fractional calculus has many definitions, such as the Riemann-Liouville type, Caputo type, Hadamard type, Erdélyi-Kober type, and Riesz type. Most of the above fractional derivatives are defined by their corresponding fractional integrals. Compared with some integral-order partial differential equations such as [9-19], fractional derivatives have hereditary and nonlocal property so that they are much more suitable for describing long-memory processes than the classical integer-order derivatives.

However, the traditional fractional calculus have complex expressions which causes many difficulties in applying them in engineering calculation, physical application, numerical modeling, etc. In addition, the traditional fractional calculus loses some basic but important properties, such as product rule and chain rule. Khalil et al. [20] proposed a new local fractional derivative, called conformable derivative, and proved the product rule and the fractional mean value theorem. Abdeljawad supplemented the Taylor power series representation, the fractional chain rule, the Gronwall inequality, and the fractional Laplace transform in [21]. Zhao et al. [22, 23] further extended the definition to the general conformable fractional derivative (GCFD). Zhang et al. defined the conformable variable order derivative in [24]. Owing to its well-behaved properties and the close connection with the classical derivatives, conformable derivative generated great research interest [25-29].

In $[30,31]$, the author considered a generalized timefractional diffusion equation by replacing the first-order time derivative to the Caputo fractional derivative. Recently, some literatures about the fractional diffusion equations with various definitions of fractional calculus occur. Al-Refai et al. $[32,33]$ considered a nonlinear fractional diffusion equation with the Riemann-Liouville time-fractional derivative and obtained their corresponding maximum principle. Borikhanov et al. [34] considered a nonlinear time-fractional diffusion equation with the Atangana-Baleanu derivative. Chen et al. [35] used the finite element method to approximate the solutions to a time-fractional advection-diffusion equations with the Caputo variable order derivative. Zhang et al. [36] obtained the sharp blow-up and global existence of solutions, a time-fractional diffusion system with the Riemann-Liouville derivative. In this paper, we consider the same equation as in [30] with the GCFD on domain $D \times(0, T), D \subset R^{n}$, i.e., 


$$
\left(D_{t}^{\alpha, \psi} u\right)(t)=-A(\mathrm{u})+F(x, t), 0<\alpha \leq 1,
$$

with the nonhomogeneous initial-boundary conditions

$$
\left\{\begin{array}{l}
\left.u\right|_{t=0}=u_{0}(x), x \in \bar{D} \\
\left.u\right|_{\partial D}=v(x, t),(x, t) \in \partial D \times[0, T]
\end{array}\right.
$$

where $F(x, t), u_{0}(x)$, and $v(x, t)$ are continuous functions, $D^{\alpha, \psi}$ is a GCFD of order $\alpha, \partial D$ is the boundary of $D$, and $-A$ is a linear elliptic operator

$$
-A(u):=p(x) \Delta u+(\nabla p, \nabla u)-q(x) u, x \in \bar{D},
$$

where $\Delta$ is the Laplace operator, $\nabla$ is the gradient operator, $p \in C^{1}(\bar{D}), q \in C(\bar{D}), p(x)>0, q(x) \geq 0$, and the domain of definition of operator $A$ is

$$
M_{A}=\left\{f, f \in C^{2}(D) \cap C^{1}(\bar{D})\right. \text {, }
$$

$f$ satisfies the boundary condition and $\left.A(f) \in L^{2}(D)\right\}$.

The GCFD is defined in the following sense (see [22]):

$$
\left(D^{\alpha, \psi} f\right)(t)=\lim _{\varepsilon \rightarrow 0} \frac{f(t+\varepsilon \psi(t, \alpha))-f(t)}{\varepsilon}, t>0,
$$

and the GCFD at 0 is defined as $\left(D^{\alpha, \psi} f\right)(0)=\lim _{t \rightarrow 0+}\left(D^{\alpha, \psi} f\right)(t)$, where $\psi(t, \alpha)$ is a continuous real function depending on $t$ and the fractional order $\alpha$ and satisfying the below conditions

$$
\begin{aligned}
& \psi(t, \alpha) \neq 0, \\
& \psi(t, 1)=1,
\end{aligned}
$$

and the relationship between the function $\psi(t, \alpha)$ and the order $\alpha$ should be one-to-one. By the definition (5), we know that the GCFD is an extension of the classical derivative $(\alpha=1$ or $\psi(t, \alpha)=1)$ and the conformable derivative $\left(\psi(t, \alpha)=t^{1-\alpha}\right)$ defined in $[20,21]$. Compared with the definition of conformable derivative in $[20,21]$ and the Definition 2.5 in [24], the GCFD in relation (5) becomes the conformable variable order derivative defined in [24] if $\psi(t, \alpha)=t^{1-\alpha}$ and the order $\alpha$ is a time-dependent function $\alpha(t)$, that is

$$
\left(D^{\alpha(t)} f\right)(t)=\lim _{\varepsilon \rightarrow 0} \frac{f\left(t+\varepsilon t^{1-\alpha(t)}\right)-f(t)}{\varepsilon}, t>0 .
$$

If the limit (5) exists, it is called that $f$ is $\alpha$-differentiable. Furthermore, if $f$ is differentiable, then by direct calculation of definition, we can obtain that $f$ is $\alpha$-differentiable and

$$
\left(D^{\alpha, \psi} f\right)(t)=f^{\prime}(t) \psi(t, \alpha)
$$

In this paper, we introduce the fractional Taylor power series expansion (see Lemma 5) and prove the theorem of term-by-term integration and differentiation (see Lemma 7 and 8) with the general conformable fractional calculus. Then by using the above and some other properties of the general conformable factional calculus (see Section 2), we obtain the maximum principle (see Theorems 9 and 10) for the classical diffusion Equation (1) with the GCFD and get some existence (see Theorems 17 and 18) and uniqueness (see Theorem 12) results of the classical solution of (1). Finally, we get the asymptotic behavior of the classical solution (see Theorem 19). The problems (1) and (2) have a solution implies that $u$ is $\alpha$-differentiable and $\left(D_{t}^{\alpha, \psi} u\right)(t)$ is continuous on $(0, T)$. We define that $u(x, t)$ is called a classical solution of problems (1) and (2), if $u(x, t) \in C(\bar{D} \times[0, T]) \cap C_{x}^{2}(D) \cap C_{t}^{1}(0, T)$ and satisfies Equation (1) and the initial-boundary condition (2).

\section{Preliminaries}

In this section, we will recall some properties of the GCFD and its fractional integral calculus.

Definition 1 (see [22]). The integral of a function $f:(0, t)$ $\longrightarrow R$ of order $\alpha$ is defined by

$$
\left(I^{\alpha, \psi} f\right)(t)=\int_{0}^{t} \frac{f(\tau)}{\psi(\tau, \alpha)} d \tau, t>0
$$

where the integral is the Riemann integral.

Remark 2. To further study the properties of general conformable fractional calculus, we assume that $\psi(t, \alpha)>0$ and $1 / \psi(\cdot, \cdot):(0, T] \times(0,1] \longrightarrow(0,+\infty)$ is Riemann integrable and this assumption is valid in the following sections.

Lemma 3 (see [20-22]). If $f, g$ is $\alpha$-differentiable at $t$, then

(1) $D^{\alpha, \psi}(c)=0$, where $c$ is any real constant,

(2) (Linearity) $D^{\alpha, \psi}(a f(t)+b g(t))=a D^{\alpha, \psi} f(t)+b D^{\alpha, \psi} g$ $(t), a, b \in R$,

(3) (Product rule) $D^{\alpha, \psi}(f(t) g(t))=f(t) D^{\alpha, \psi} g(t)+g(t)$ $D^{\alpha, \psi} f(t)$, $D^{\alpha, \psi}(f(t) / g(t))=g(t) D^{\alpha, \psi} f(t)-f(t) D^{\alpha, \psi} g(t) / g^{2}(t)$,

(4) (Chain rule) If $f$ is $\alpha$-differentiable at $g$ and $g \neq 0$, then

$$
D^{\alpha, \psi}(f(g(t)))=\left(D^{\alpha, \psi} f\right)(g(t)) \cdot D^{\alpha, \psi}(g(t)) \cdot \frac{1}{\psi(g(t), \alpha)}
$$

If $f$ is differentiable at $g$, then $D^{\alpha, \psi}(f(g(t)))=f^{\prime}(g(t))$ $D^{\alpha, \psi}(g(t))$. 
Remark 4. Especially, by the definition (5), the relation (8) and Lemma 3 (4), we have

$$
D^{\alpha, \psi}\left(\int_{0}^{t} \frac{1}{\psi(\tau, \alpha)} d \tau\right)=1, D^{\alpha, \psi}\left(e^{\int_{0}^{t} \frac{1}{\psi(\tau, \alpha)} d \tau}\right)=e^{\int_{0}^{t} \frac{1}{\psi(\tau, \alpha)} d \tau},
$$

which coincides with the classical derivatives $(\psi(t, \alpha)=1)$ and the conformable fractional derivatives $\left(\psi(t, \alpha)=t^{1-\alpha}\right)$ defined in $[20,21]$.

Lemma 5 (Fractional Taylor power series expansions). Assume that $f$ is an infinite $\alpha$-differentiable function at a neighbourhood of $t_{0}$. Then $f$ has the fractional power series expansion at point $t_{0}$ :

$f(t)=\sum_{k=0}^{+\infty} \frac{\left(D^{\alpha, \psi} f\right)^{(k)}\left(t_{0}\right)}{k !}\left(\int_{t_{0}}^{t} \frac{1}{\psi(\tau, \alpha)} d \tau\right)^{k}, t_{0}<t<t_{0}+R, R>0$,

where $\left(D^{\alpha, \psi} f\right)^{(k)}\left(t_{0}\right)$ means the application of the GCFD $k$ times.

Proof. The proof is similar to that of Theorem 17 in [21]. And this result coincides with the classical derivatives and the conformable derivatives. Especially, the exponential function $e^{\int_{t_{0}}^{t}(1 / \psi(\tau, \alpha)) d \tau}$ has the factional Taylor power series expansion at point $t_{0}$ :

$$
e^{\int_{t_{0}}^{t} \frac{1}{\psi(\tau, \alpha)} d \tau}=\sum_{k=0}^{+\infty} \frac{1}{k !}\left(\int_{t_{0}}^{t} \frac{1}{\psi(\tau, \alpha)} d \tau\right)^{k} .
$$

Lemma 6 (see [22]). Let $f$ be a continuous differentiable function on $(0, T)$, then

$$
I^{\alpha, \psi}\left(D^{\alpha, \psi} f(t)\right)=f(t)-f(0), D^{\alpha, \psi}\left(I^{\alpha, \psi} f(t)\right)=f(t) .
$$

Lemma 7. Let a sequence of functions $f_{i}(t), i=1,2, \cdots$ satisfying the following conditions:

(1) for any given $\alpha(>0)$, there exists fractional integrals $\left(I^{\alpha, \psi} f_{i}\right)(t), i=1,2, \cdots$

(2) both the series $\sum_{i=1}^{+\infty} f_{i}(t)$ and $\sum_{i=1}^{+\infty}\left(I^{\alpha, \psi} f_{i}\right)(t)$ are uniformly convergent for any $t \in[0, T]$ then the function $\sum_{i=1}^{+\infty} f_{i}(t)$ is $\alpha$-integrable on $(0, T)$ and

$$
I^{\alpha, \psi}\left(\sum_{i=1}^{+\infty} f_{i}(t)\right)=\sum_{i=1}^{+\infty}\left(I^{\alpha, \psi} f_{i}\right)(t)
$$

Proof. Due to the uniformly convergence of $\sum_{i=1}^{+\infty} f_{i}(t)$ and $\sum_{i=1}^{+\infty}\left(I^{\alpha, \psi} f_{i}\right)(t)$, then for any $\varepsilon>0$, there exists a positive integer $N$ such that for any $n>N$ and $t \in(0, T)$,

$$
\left|\sum_{i=1}^{+\infty} f_{i}(t)-\sum_{i=1}^{n} f_{i}(t)\right|<\frac{\varepsilon}{2 \int_{0}^{T}(1 / \psi(\tau, \alpha)) d \tau},\left|\sum_{i=n}^{+\infty}\left(I^{\alpha, \psi} f_{i}\right)(t)\right|<\frac{\varepsilon}{2} .
$$

Therefore, by (16) and the linearity of the operator $I^{\alpha, \psi}$, we obtain

$$
\begin{aligned}
& \left|I^{\alpha, \psi}\left(\sum_{i=1}^{+\infty} f_{i}(t)\right)-\sum_{i=1}^{+\infty}\left(I^{\alpha, \psi} f_{i}\right)(t)\right| \\
& \quad \leq\left|I^{\alpha, \psi}\left(\sum_{i=1}^{+\infty} f_{i}(t)-\sum_{i=1}^{n} f_{i}(t)\right)\right|+\left|\sum_{i=n+1}^{+\infty}\left(I^{\alpha, \psi} f_{i}\right)(t)\right| \\
& \quad \leq\left|\int_{0}^{t} \frac{\sum_{i=1}^{+\infty} f_{i}(\tau)-\sum_{i=1}^{n} f_{i}(\tau)}{\psi(\tau, \alpha)} d \tau\right|+\left|\sum_{i=n+1}^{+\infty}\left(I^{\alpha, \psi} f_{i}\right)(t)\right|<\varepsilon .
\end{aligned}
$$

i.e., the function $\sum_{i=1}^{+\infty} f_{i}(t)$ is $\alpha$-integrable and $I^{\alpha, \psi}\left(\sum_{i=1}^{+\infty}\right.$ $\left.f_{i}(t)\right)=\sum_{i=1}^{+\infty}\left(I^{\alpha, \psi} f_{i}\right)(t)$, then the proof is completed.

Lemma 8. Let a continuous differentiable sequence of functions $f_{i}(t), i=1,2, \cdots$ satisfying the following conditions:

(1) for any given $\alpha(>0)$, there exists fractional integrals $\left(D^{\alpha, \psi} f_{i}\right)(t), i=1,2, \cdots$

(2) both the series $\sum_{i=1}^{+\infty} f_{i}(t)$ and $\sum_{i=1}^{+\infty}\left(D^{\alpha, \psi} f_{i}\right)(t)$ are uniformly convergent for any $t \in[0, T]$. Moreover, $\sum_{i=1}^{+\infty}\left(D^{\alpha, \psi} f_{i}\right)(t) \in L(0, T)$, then the function $\sum_{i=1}^{+\infty}$ $f_{i}(t)$ is $\alpha$-differentiable on $(0, T)$ and

$$
D^{\alpha, \psi}\left(\sum_{i=1}^{+\infty} f_{i}(t)\right)=\sum_{i=1}^{+\infty}\left(D^{\alpha, \psi} f_{i}\right)(t)
$$

Proof. Since $\sum_{i=1}^{+\infty} f_{i}(t)$ and $\sum_{i=1}^{+\infty}\left(D^{\alpha, \psi} f_{i}\right)(t)$ are uniformly convergent for any $t \in[0, T]$, then by Lemma 6 and Lemma 7 .

$$
\begin{aligned}
I^{\alpha, \psi}\left(\sum_{i=1}^{+\infty}\left(D^{\alpha, \psi} f_{i}\right)(t)\right) & =\sum_{i=1}^{+\infty}\left(I^{\alpha, \psi}\left(\left(D^{\alpha, \psi} f_{i}\right)(t)\right)\right) \\
& =\sum_{i=1}^{+\infty} f_{i}(t)-\sum_{i=1}^{+\infty} f_{i}(0) .
\end{aligned}
$$

The left side of the above equality is $\alpha$-differentiable; then by Lemma 3(1), $\sum_{i=1}^{+\infty} f_{i}(t)$ is $\alpha$-differentiable and

$$
\begin{aligned}
D^{\alpha, \psi}\left(\sum_{i=1}^{+\infty} f_{i}(t)\right) & =D^{\alpha, \psi}\left(\sum_{i=1}^{+\infty} f_{i}(t)-\sum_{i=1}^{+\infty} f_{i}(0)\right) \\
& =\sum_{i=1}^{+\infty}\left(D^{\alpha, \psi} f_{i}\right)(t) .
\end{aligned}
$$




\section{The Uniqueness of Solution}

Theorem 9. Let $u$ be a classical solution of Equation (1) in the domain $D \times(0, T)$ and $F(x, t) \leq 0$, then

$$
u(x, t) \leq \max \left\{0, \max _{(x, t) \in S} u(x, t)\right\},(x, t) \in \bar{D} \times[0, T]
$$

where $S:=(\bar{D} \times\{0\}) \cup(\partial D \times[0, T])$.

Proof. The proof follows by Lemma 3 (4) and setting the same auxiliary function $w(x, t):=u(x, t)+(\varepsilon / 2) \cdot(T-t / T)$ as [30]. Due to $u$ is a classical solution of the problems (1) and (2), then $u$ is differentiable with respect to $t$. Therefore, we have $\left(D_{t}^{\alpha, \psi} u\right)\left(t_{0}\right)=\psi\left(t_{0}, \alpha\right) u_{t}\left(x_{0}, t_{0}\right)=0$, where $\left(x_{0}, t_{0}\right)$ is the maximum point of $u$ over the domain $\bar{D} \times[0, T]$.

Theorem 10. Let $u$ be a classical solution of Equation (1) in the domain $D \times(0, T)$ and $F(x, t) \geq 0, \quad(x, t) \in D \times(0, T)$, then

$$
u(x, t) \geq \min _{(x, t) \in S} u(x, t),(x, t) \in \bar{D} \times[0, T]
$$

Theorem 11. Let $u$ be a classical solution of problems (1) and (2) and $F \in C(\bar{D} \times[0, T])$ with the norm $\|F\|_{C(\bar{D} \times[0, T])}=$ $\sup _{\bar{D} \times[0, T]}|F(x, t)|:=M_{1}$.

Denote by $\quad M_{2}=\left\|u_{0}\right\|_{C(\bar{D})}=\sup _{\bar{D}}\left|u_{0}(x)\right|, \quad M_{3}=$ $\|v\|_{C(\partial D \times[0, T])}=\sup _{\partial D \times[0, T]}|v(x, t)|$, then the following estimate of the solution norm holds true:

$$
\|u\|_{C(\bar{D} \times[0, T])} \leq 2 C M_{1}+\max \left\{M_{2}, M_{3}\right\},
$$

where $C:=\int_{0}^{T}(1 / \psi(\tau, \alpha)) d \tau$.

Proof. Define the auxiliary function $g$ :

$$
g(x, t)=u(x, t)-\int_{0}^{t} \frac{M_{1}}{\psi(\tau, \alpha)} d \tau,(x, t) \in \bar{D} \times[0, T]
$$

By the continuity of $\psi$ and Remark 4 , then $g$ is a classical solution of the following problem:

$$
\left\{\begin{array}{l}
\left(D_{t}^{\alpha, \psi} g\right)(t)=-A(g)+F_{1}(x, t),(x, t) \in D \times(0, T), \\
\left.g\right|_{t=0}=u_{0}, x \in \bar{D} \\
\left.g\right|_{\partial D}=v(x, t)-\int_{0}^{t} \frac{M_{1}}{\psi(\tau, \alpha)} d \tau:=v_{1}(x, t),(x, t) \in \partial D \times[0, T],
\end{array}\right.
$$

where $\quad F_{1}(x, t)=F(x, t)-M_{1}-q(x) \int_{0}^{t}\left(M_{1} / \psi(\tau, \alpha)\right) d \tau \leq 0$. According to the conditions, we have

$$
\left\|v_{1}\right\|_{C(\partial D \times[0, T])} \leq M_{3}+C M_{1}
$$

Then by Theorem 9, the classical solution $g$ satisfies the estimate,

$$
g(x, t) \leq \max \left\{M_{2}, M_{3}+C M_{1}\right\},(x, t) \in \bar{D} \times[0, T],
$$

which means that

$$
u(x, t) \leq 2 C M_{1}+\max \left\{M_{2}, M_{3}\right\} .
$$

Similarly, consider of the auxiliary function $g_{1}$ and using the Theorem 10

$$
g_{1}(x, t)=u(x, t)+\int_{0}^{t} \frac{M_{1}}{\psi(\tau, \alpha)} d \tau,(x, t) \in \bar{D} \times[0, T]
$$

then we have

$$
g_{1}(x, t) \geq \max \left\{-M_{2},-M_{3}-C M_{1}\right\},(x, t) \in \bar{D} \times[0, T],
$$

i.e., $u(x, t) \geq-2 C M_{1}-\max \left\{M_{2}, M_{3}\right\}$. Combined with (28), we obtain

$$
\|u\|_{C(\bar{D} \times[0,+\infty))} \leq 2 C M_{1}+\max \left\{M_{2}, M_{3}\right\} .
$$

Theorem 12. The problems (1) and (2) have at most one classical solution and the solution continuously depends on the data given in the problem in the sense that if

$$
\begin{aligned}
\left\|F_{1}-F_{2}\right\|_{C(\bar{D} \times[0, T])} & \leq \varepsilon_{1},\left\|u_{0}^{(1)}-u_{0}^{(2)}\right\|_{C(\bar{D})} \\
& \leq \varepsilon_{2},\left\|v_{1}-v_{2}\right\|_{C(\partial D \times[0, T])} \leq \varepsilon_{3},
\end{aligned}
$$

then for the corresponding classical solution $u_{1}$ and $u_{2}$, the estimate holds:

$$
\left\|u_{1}-u_{2}\right\|_{C(\bar{D} \times[0, T])} \leq 2 C \varepsilon_{1}+\max \left\{\varepsilon_{2}, \varepsilon_{3}\right\}
$$

Proof. Let $u_{1}, u_{2}$ be the classical solution of the following problem, respectively,

$$
\left\{\begin{array}{l}
\left(D_{t}^{\alpha, \psi} u\right)(t)=-A(u)+F_{i}(x, t),(x, t) \in D \times(0, T), \\
\left.u\right|_{t=0}=u_{0}^{(i)}(t), x \in \bar{D} \\
\left.u\right|_{\partial D}=v_{i}(x, t),(x, t) \in \partial D \times[0, T]
\end{array}\right.
$$

where $i=1,2$. Then $u=u_{1}-u_{2}$ is the classical solution of the corresponding problem 


$$
\left\{\begin{array}{l}
\left(D_{t}^{\alpha, \psi} u\right)(t)=-A(u)+F_{1}(x, t)-F_{2}(x, t),(x, t) \in D \times(0, T), \\
\left.u\right|_{t=0}=u_{0}^{(1)}(t)-u_{0}^{(2)}(t), x \in \bar{D}, \\
\left.u\right|_{\partial D}=v_{1}(x, t)-v_{2}(x, t),(x, t) \in \partial D \times[0, T] .
\end{array}\right.
$$

By (23), the estimate (33) holds. The uniqueness of the classical solution of (1) and (2) is a direct consequence of Theorem 9, Theorem 10 and the estimate (33).

\section{The Existence of Solution}

In this section, we will consider some existence results of solution of problems (1) and (2) with homogenous boundary conditions

$$
\left\{\begin{array}{l}
\left.u\right|_{t=0}=u_{0}(x), x \in \bar{D}, \\
\left.u\right|_{\partial D}=0,(x, t) \in \partial D \times[0, T] .
\end{array}\right.
$$

Firstly, we consider the homogenous equation, i.e., $F(x, t) \equiv 0$. In the following, we seek the solution of Equation (1) by using the variable separation method in the form

$$
u(x, t)=X(x) T(t),(x, t) \in D \times(0, T),
$$

which satisfies the boundary condition. Substituting expression (37) in Equation (1) and separating the variables, we obtain the following sense:

$$
\frac{\left(D^{\alpha, \psi} T\right)(t)}{T(t)}=-\frac{A(X(x))}{X(x)}=-\lambda,
$$

where $\lambda$ is a constant independent on $t$ and $x$. Therefore, addition to the boundary condition, we have

$$
\left(D^{\alpha, \psi} T\right)(t)+\lambda T(t)=0,
$$

and the eigenvalues problem for the operator $A$

$$
\left\{\begin{array}{l}
A(X(x))=\lambda X(x),(x, t) \in D \times(0, T), \\
\left.X\right|_{\partial D}=0,(x, t) \in \partial D \times[0, T] .
\end{array}\right.
$$

The properties of $A$ is well known in [37] that $A$ is a positive and self-adjoint linear operator. Moreover, (40) has a counted number of positive eigenvalues $0<\lambda_{1} \leq \lambda_{2} \leq \cdots, \lambda_{k}$ $\longrightarrow+\infty(k \longrightarrow+\infty)$ with finite multiplicity, and any function $f \in M_{A}$ can be represented through its Fourier series:

$$
f(x)=\sum_{i=1}^{+\infty}\left(f, X_{i}\right) X_{i}(x),
$$

where $X_{i} \in M_{A}$ is the corresponding eigenfunction of the eigenvalue $\lambda_{i}$. In fact, in the following, we choose $\left\{X_{i}\right\}_{i=1}^{+\infty}$ to be real and orthonormal.
For Equation (39) and its inhomogenous equation

$$
\left(D^{\alpha, \psi} T\right)(t)+\lambda T(t)=f(t),
$$

we consider the following first-order linear differential equation for $\lambda=\lambda_{i}$ :

$$
T_{i}^{\prime}(t)+\frac{\lambda_{i}}{\psi(t, \alpha)} T_{i}(t)=\frac{f(t)}{\psi(t, \alpha)} .
$$

It is well known that the solution of Equation (43) is given by

$$
T_{i}(t)=e^{-\int_{0}^{t} \frac{\lambda_{i}}{\psi(\tau, \alpha)} d \tau}\left[c_{i}+\int_{0}^{t} e_{0}^{\tau} \frac{\lambda_{i}}{\psi(s, \alpha)} d s \frac{f(\tau)}{\psi(\tau, \alpha)} d \tau\right],
$$

where $c_{i}$ are some constants. Obviously, $T_{i}(t) \in C[0, T] \cap C^{1}$ $(0, T)$. Moreover, $T_{i}(t)$ in (44) satisfies the following equation:

$$
\psi(\tau, \alpha) T_{i}^{\prime}(t)+\lambda_{i} T_{i}(t)=f(t) .
$$

Hence, according to the relation (8) we have

$$
\left(D^{\alpha, \psi} T_{i}\right)(t)+\lambda_{i} T_{i}(t)=\psi(\tau, \alpha) T_{i}^{\prime}(t)+\lambda_{i} T_{i}(t)=f(t),
$$

which means the function in (44) is the solution of Equation (42). Correspondingly, the solution of Equation (39) for $\lambda=\lambda_{i}$ is

$$
T_{i}(t)=c_{i} e^{-\int_{0}^{t} \frac{\lambda_{i}}{\psi(\tau, \alpha)} d \tau}
$$

Naturally, each function

$$
T_{i}(t) X_{i}(x)=c_{i} e^{-\int_{0}^{t} \frac{\lambda_{i}}{\psi(\tau, \alpha)} d \tau} X_{i}(x),
$$

and its finite sum

$$
u_{k}(x, t)=\sum_{i=1}^{k} T_{i}(t) X_{i}(x)=\sum_{i=1}^{k} c_{i} e^{-\int_{0}^{t} \frac{\lambda_{i}}{\psi(\tau, \alpha)} d \tau} X_{i}(x)
$$

satisfy Equation (1) and the boundary condition.

In addition to the initial condition, we define the formal solution of problems (1) and (36) as the following sense:

$$
u(x, t)=\sum_{i=1}^{+\infty} T_{i}(t) X_{i}(x)=\sum_{i=1}^{+\infty}\left(u_{0}, X_{i}\right) e^{-\int_{0}^{t} \frac{\lambda_{i}}{\psi(\tau, \alpha)} d \tau} X_{i}(x) .
$$

Next, we consider the case of inhomogenous equation. Similarly to the case of homogenous condition, the formal solution of inhomogenous equation also has the expression (50) with appropriate $T_{i}(t)$. For any $i$, we multiply Equation (1) by $X_{i}$, integrating over $D$. Then by Lemma 8 and using the 
self-adjoint property of the operator $A$, combined by the orthogonality of $\left\{X_{i}\right\}_{i=1}^{+\infty}$, we obtain

$$
\begin{aligned}
\left(D^{\alpha, \psi} T_{i}\right)(t) & =\int_{D}\left(D_{t}^{\alpha, \psi} u\right)(t) \cdot X_{i}(x) d x \\
& =\int_{D} D_{t}^{\alpha, \psi}\left(\sum_{i=1}^{+\infty} T_{i}(t) X_{i}(x)\right) \cdot X_{i}(x) d x \\
& =\int_{D}\left(\sum_{i=1}^{+\infty} D_{t}^{\alpha, \psi}\left(T_{i}(t) X_{i}(x)\right)\right) \cdot X_{i}(x) d x \\
& =-\left(A u, X_{i}\right)+\left(F, X_{i}\right)=-\lambda_{i}\left(u, X_{i}\right)+\left(F, X_{i}\right) \\
& =-\lambda_{i} T_{i}+\left(F, X_{i}\right) .
\end{aligned}
$$

That is

$$
\left(D^{\alpha, \psi} T_{i}\right)(t)+\lambda_{i} T_{i}(t)=\left(F(x, t), X_{i}(x)\right) .
$$
(52) is

$$
T_{i}(t)=e^{-\int_{0}^{t} \frac{\lambda_{i}}{\psi(\tau, \alpha)} d \tau}\left[c_{i}+\int_{0}^{t} e^{\int_{0}^{\tau} \frac{\lambda_{i}}{\psi(s, \alpha)} d s} \frac{\left(F(x, \tau), X_{i}(x)\right)}{\psi(\tau, \alpha)} d \tau\right]
$$

where $c_{i}$ are some constants. Consequently, combined with the initial condition, we define the formal solution of problems (1) and (36) as the following sense:

$$
\begin{aligned}
u(x, t)= & \sum_{i=1}^{+\infty} T_{i}(t) X_{i}(x)=\sum_{i=1}^{+\infty}\left(u_{0}, X_{i}\right) e^{-\int_{0}^{t} \frac{\lambda_{i}}{\psi(\tau, \alpha)} d \tau} X_{i}(x) \\
& +\sum_{i=1}^{+\infty} e^{-\int_{0}^{t} \frac{\lambda_{i}}{\psi(\tau, \alpha)} d \tau} \int_{0}^{t} e^{\int_{0}^{\tau} \frac{\lambda_{i}}{\psi(s, \alpha)} d s} \frac{\left(F(x, \tau), X_{i}(x)\right) X_{i}(x)}{\psi(\tau, \alpha)} d \tau
\end{aligned}
$$

Remark 13. For Equation (52), we try applying the operator $I^{\alpha, \psi}$ to its both sides, then by Definition 1 and Lemma 6, we have

$$
T_{i}(t)=T_{i}(0)-\lambda_{i} \int_{0}^{t} \frac{T_{i}(\tau)}{\psi(\tau, \alpha)} d \tau+\int_{0}^{t} \frac{\left(F(x, \tau), X_{i}(x)\right)}{\psi(\tau, \alpha)} d \tau
$$

Next, we apply the method of successive approximations to solve this integral equation by setting

$$
\begin{aligned}
T_{i}^{(0)}(t)= & T_{i}(0), \\
T_{i}^{(m)}(t)= & T_{i}^{(0)}(t)-\lambda_{i} \int_{0}^{t} \frac{T_{i}^{(m-1)}(\tau)}{\psi(\tau, \alpha)} d \tau \\
& +\int_{0}^{t} \frac{\left(F(x, \tau), X_{i}(x)\right)}{\psi(\tau, \alpha)} d \tau, m \in N^{+} .
\end{aligned}
$$

Taking (56) into account, then

$$
T_{i}^{(1)}(t)=T_{i}(0)-\lambda_{i} T_{i}(0) \int_{0}^{t} \frac{1}{\psi(\tau, \alpha)} d \tau+\int_{0}^{t} \frac{\left(F(x, \tau), X_{i}(x)\right)}{\psi(\tau, \alpha)} d \tau
$$

Similarly, using (56)-(58), we have

$$
\begin{aligned}
T_{i}^{(2)}(t)= & T_{i}(0)-\lambda_{i} T_{i}(0) \int_{0}^{t} \frac{1}{\psi(\tau, \alpha)} d \tau+\lambda_{i}^{2} T_{i}(0) \int_{0}^{t} \int_{0}^{s} \frac{1}{\psi(\tau, \alpha)} \\
& \cdot \frac{1}{\psi(s, \alpha)} d s d \tau+\int_{0}^{t} \frac{\left(F(x, \tau), X_{i}(x)\right)}{\psi(\tau, \alpha)}\left[1-\lambda_{i} \int_{\tau}^{t} \frac{1}{\psi(s, \alpha)} d s\right] d \tau \\
= & \sum_{k=0}^{2} \frac{\left(-\lambda_{i}\right)^{k} T_{i}(0)}{k !}\left(\int_{0}^{t} \frac{1}{\psi(\tau, \alpha)} d \tau\right)^{k} \\
& +\int_{0}^{t} \frac{\left(F(x, \tau), X_{i}(x)\right)}{\psi(\tau, \alpha)}\left[\sum_{k=0}^{1} \frac{\left(-\lambda_{i}\right)^{k}}{k !}\left(\int_{\tau}^{t} \frac{1}{\psi(\tau, \alpha)} d s\right)^{k}\right] d \tau .
\end{aligned}
$$

Continuing this process, we obtain the following expression for $T_{i}^{(m)}(t)$ :

$$
\begin{aligned}
T_{i}^{(m)}(t)= & \sum_{k=0}^{m} \frac{\left(-\lambda_{i}\right)^{k} T_{i}(0)}{k !}\left(\int_{0}^{t} \frac{1}{\psi(\tau, \alpha)} d \tau\right)^{k} \\
& +\int_{0}^{t} \frac{\left(F(x, \tau), X_{i}(x)\right)}{\psi(\tau, \alpha)}\left[\sum_{k=0}^{m-1} \frac{\left(-\lambda_{i}\right)^{k}}{k !}\left(\int_{\tau}^{t} \frac{1}{\psi(\tau, \alpha)} d s\right)^{k}\right] d \tau
\end{aligned}
$$

Taking the limit as $m \longrightarrow+\infty$, we obtain

$$
\begin{aligned}
T_{i}(t)= & \sum_{k=0}^{+\infty} \frac{\left(-\lambda_{i}\right)^{k} T_{i}(0)}{k !}\left(\int_{0}^{t} \frac{1}{\psi(\tau, \alpha)} d \tau\right)^{k} \\
& +\int_{0}^{t} \frac{\left(F(x, \tau), X_{i}(x)\right)}{\psi(\tau, \alpha)}\left[\sum_{k=0}^{+\infty} \frac{\left(-\lambda_{i}\right)^{k}}{k !}\left(\int_{\tau}^{t} \frac{1}{\psi(\tau, \alpha)} d s\right)^{k}\right] d \tau .
\end{aligned}
$$

By Lemma 5, the expression (61) is the fractional Taylor power series expansion of (53).

Definition 14. A formal solution of the problems (1) and $((36))$ is called the Fourier series in the form $(50)(F \equiv 0)$ and $(54)(F \neq 0)$.

Definition 15. Let the sequences of $u_{0 k} \in C(\bar{D})$ and $v_{k} \in C$ $(\partial D \times[0, T]), \quad F_{k} \in C(\bar{D} \times[0, T]), \quad k=1,2, \cdots$ such that for $k \longrightarrow+\infty, u_{0 k} \longrightarrow u_{0}, v_{k} \longrightarrow v, F_{k} \longrightarrow F$ in $L^{2}(D)$ and $t \in[0, T]$. And for any $k=1,2, \cdots$, there exists a classical solution $u_{k}$ of the following problem

$$
\left\{\begin{array}{l}
\left(D_{t}^{\alpha, \psi} u_{k}\right)(t)=-A\left(u_{k}\right)+F_{k}(x, t), \\
\left.u_{k}\right|_{t=0}=u_{0 k}(x), x \in \bar{D}, \\
\left.u_{k}\right|_{\partial D}=v_{k}(x, t),(x, t) \in \partial D \times[0, T] .
\end{array}\right.
$$


Suppose there exists a function $u \in C(\bar{D} \times[0, T])$ such that $u_{k} \longrightarrow u$ as $k \longrightarrow+\infty$, then the function $u$ is called a generalized solution of the problems (1) and (2).

Remark 16. By Theorem 12, the convergence of $u_{0 k}, v_{k}, F_{k}$ and the completeness of $C\left(\bar{D} \times[0, T],\|\cdot\|_{C(\bar{D} \times[0, T])}\right)$, we know that there always exists a function $u \in C(\bar{D} \times[0, T])$ which is the limit of the sequence $u_{k}$. In fact, under the assumption $u_{0}$, $F \in M_{A}$, the formal solution is also the generalized solution of problems (1) and (36). That is the following theorem.

Theorem 17. Suppose that $u_{0}, F \in M_{A}$, then the formal solution (54) is the generalized solution of the problems (1) and (36).

Proof. For every $k=1,2, \cdots$,

$$
\begin{aligned}
u_{k}(x, t)= & \sum_{i=1}^{k}\left(u_{0}, X_{i}\right) e^{-\int_{0}^{t} \frac{\lambda_{i}}{\psi(\tau, \alpha)} d \tau} X_{i}(x) \\
& +\sum_{i=1}^{k} e^{-\int_{0}^{t} \frac{\lambda_{i}}{\psi(\tau, \alpha)} d \tau} \int_{0}^{t} e_{0}^{\tau} \frac{\lambda_{i}}{\psi(s, \alpha)} d s \frac{\left(F(x, \tau), X_{i}(x)\right) X_{i}(x)}{\psi(\tau, \alpha)} d \tau,
\end{aligned}
$$

is the classical solution of the problems (1) and (36) with the initial condition

$$
u_{0 k}(x)=\sum_{i=1}^{k}\left(u_{0}, X_{i}\right) X_{i}(x)
$$

Note that $u_{0} \in M_{A}, u_{0 k} \longrightarrow u_{0}$ as $k \longrightarrow+\infty$ in $\bar{D} \times[0, T]$ and $e^{-\int_{0}^{t}\left(\lambda_{i} / \psi(\tau, \alpha)\right) d \tau} \leq 1$; therefore, $\sum_{i=1}^{k}\left(u_{0}, X_{i}\right) e^{-\int_{0}^{t}\left(\lambda_{i} / \psi(\tau, \alpha)\right) d \tau}$ $X_{i}(x)$ uniformly converges on $\bar{D} \times[0, T]$.

In view of $F \in M_{A}$, then $F(x, t)=\sum_{i=1}^{+\infty}\left(F(x, t), X_{i}\right) X_{i}(x)$ uniformly converges on $\bar{D} \times[0, T]$. Therefore,

$$
\begin{aligned}
& \sum_{i=1}^{k} e^{-\int_{0}^{t} \frac{\lambda_{i}}{\psi(\tau, \alpha)} d \tau} \int_{0}^{t} e_{0}^{\tau} \frac{\lambda_{i}}{\psi(s, \alpha)} d s\left|\frac{\left(F(x, \tau), X_{i}(x)\right) X_{i}(x)}{\psi(\tau, \alpha)}\right| d \tau \\
& \quad \leq \int_{0}^{t}\left|\frac{1}{\psi(\tau, \alpha)} \cdot\left(\sum_{i=1}^{k}\left(F(x, \tau), X_{i}\right) X_{i}(x)\right)\right| d \tau \\
& \quad \longrightarrow \int_{0}^{t}\left|\frac{1}{\psi(\tau, \alpha)} \cdot\left(\sum_{i=1}^{+\infty}\left(F(x, \tau), X_{i}\right) X_{i}(x)\right)\right| d \tau(\text { ask } \longrightarrow+\infty) .
\end{aligned}
$$

Consequently, (54) is the generalized solution of the problems (1) and (36).

Theorem 18. Suppose that $u_{0}, F \in M_{A}, A\left(u_{0}\right), A^{2}\left(u_{0}\right) \in M_{A}$ and for any $t \in(0, T), A(F), A^{2}(F) \in M_{A}$, then the formal solution (54) is the classical solution of the problems (1) and (36).

Proof. By Theorem 17, the formal solution (54) is the generalized solution; it remains to prove that (54) is at least twice differentiable with respect to the spatial variable $x$ and differentiable with respect to the time variable $t$.

Differentiating term-by-term (54) with respect to $x$, we construct a series

$$
\begin{aligned}
& \sum_{i=1}^{+\infty}\left(u_{0}, X_{i}\right) e^{-\int_{0}^{t} \frac{\lambda_{i}}{\psi(\tau, \alpha)} d \tau} \nabla X_{i}(x) \\
& \quad+\sum_{i=1}^{+\infty} e^{-\int_{0}^{t} \frac{\lambda_{i}}{\psi(\tau, \alpha)} d \tau} \int_{0}^{t} e^{\int_{0}^{\tau} \frac{\lambda_{i}}{\psi(s, \alpha)} d s} \frac{\left(F(x, \tau), X_{i}(x)\right) \nabla X_{i}(x)}{\psi(\tau, \alpha)} d \tau .
\end{aligned}
$$

Now we shall prove that the above series (66) is uniformly convergent. Since $u_{0} \in M_{A}, F \in M_{A}$, then $A\left(u_{0}\right)$, $A(F) \in L^{2}(D)$. Moreover, $A\left(u_{0}\right), A(F) \in M_{A}$, so $A\left(u_{0}\right)$ and $A(F)$ can be expanded into uniformly convergent series

$$
A\left(u_{0}\right)=\sum_{i=1}^{+\infty}\left(A\left(u_{0}\right), X_{i}\right) X_{i}, A(F)=\sum_{i=1}^{+\infty}\left(A(F), X_{i}\right) X_{i},
$$

for any $t \in(0, T)$. Then by the Parseval equality,

$$
\begin{aligned}
\left\|A\left(u_{0}\right)\right\|_{2}^{2} & =\sum_{i=1}^{+\infty}\left|\left(A\left(u_{0}\right), X_{i}\right)\right|^{2}=\sum_{i=1}^{+\infty}\left|\left(u_{0}, A\left(X_{i}\right)\right)\right|^{2} \\
& =\sum_{i=1}^{+\infty} \lambda_{i}^{2}\left|\left(u_{0}, X_{i}\right)\right|^{2}<+\infty, \\
\|A(F)\|_{2}^{2} & =\sum_{i=1}^{+\infty}\left|\left(A(F), X_{i}\right)\right|^{2}=\sum_{i=1}^{+\infty}\left|\left(F, A\left(X_{i}\right)\right)\right|^{2} \\
& =\sum_{i=1}^{+\infty} \lambda_{i}^{2}\left|\left(F, X_{i}\right)\right|^{2}<+\infty .
\end{aligned}
$$

Considering that the series $\sum_{i=1}^{+\infty}\left(\left|\nabla X_{i}\right|^{2} / \lambda_{i}^{2}\right)$ uniformly convergent (see [38]), then applying the Cauchy inequality we have

$$
\begin{aligned}
\sum_{i=1}^{+\infty}\left|\left(F(x, t), X_{i}(x)\right) \nabla X_{i}(x)\right| \leq & \sum_{i=1}^{+\infty}\left|\lambda_{i}\left(F(x, t), X_{i}(x)\right)\right| \cdot\left|\frac{\nabla X_{i}(x)}{\lambda_{i}}\right| \\
\leq & \left(\sum_{i=1}^{+\infty} \lambda_{i}^{2}\left|\left(F(x, t), X_{i}(x)\right)\right|^{2}\right)^{1 / 2} \\
& \cdot\left(\sum_{i=1}^{+\infty}\left(\frac{\left|\nabla X_{i}(x)\right|^{2}}{\lambda_{i}^{2}}\right)\right)^{1 / 2} \\
\leq & \|A(F)\|_{2}\left(\sum_{i=1}^{+\infty}\left(\frac{\left|\nabla X_{i}(x)\right|^{2}}{\lambda_{i}^{2}}\right)\right)^{1 / 2},
\end{aligned}
$$

which implies that the series $\sum_{i=1}^{+\infty}\left|\left(F(x, t), X_{i}(x)\right) \nabla X_{i}(x)\right|$ is uniformly convergent on $D$ for any $t \in(0, T)$. In view that $\left\{X_{i}\right\}_{i=1}^{+\infty}$ is a complete orthonormal system in $L^{2}(D)$, using 
the above facts, the estimates (68)-(70) and applying the Cauchy inequality, we get

$$
\begin{aligned}
& \sum_{i=1}^{+\infty}\left|\left(u_{0}, X_{i}\right) e^{-\int_{0}^{t} \frac{\lambda_{i}}{\psi(\tau, \alpha)} d \tau} \nabla X_{i}(x)\right| \\
& +\sum_{i=1}^{+\infty}\left|e^{-\int_{0}^{t} \frac{\lambda_{i}}{\psi(\tau, \alpha)} d \tau} \int_{0}^{t} e^{\int_{0}^{\tau} \frac{\lambda_{i}}{\psi(s, \alpha)} d s} \frac{\left(F(x, \tau), X_{i}(x)\right) \nabla X_{i}(x)}{\psi(\tau, \alpha)} d \tau\right| \\
& \leq \sum_{i=1}^{+\infty}\left|\left(u_{0}, X_{i}\right) \nabla X_{i}(x)\right| \\
& +\sum_{i=1}^{+\infty}\left|\int_{0}^{t} \frac{\left(F(x, \tau), X_{i}(x)\right) \nabla X_{i}(x)}{\psi(\tau, \alpha)} d \tau\right| \\
& \leq \sum_{i=1}^{+\infty}\left|\lambda_{i}\left(u_{0}, X_{i}\right)\right| \cdot\left|\frac{\nabla X_{i}(x)}{\lambda_{i}}\right| \\
& +\int_{0}^{t} \frac{1}{\psi(\tau, \alpha)}\left(\sum_{i=1}^{+\infty}\left|\lambda_{i}\left(F(x, \tau), X_{i}(x)\right)\right| \cdot\left|\frac{\nabla X_{i}(x)}{\lambda_{i}}\right|\right) d \tau \\
& \leq\left(\sum_{i=1}^{+\infty} \lambda_{i}^{2}\left(u_{0}, X_{i}\right)^{2}\right)^{1 / 2} \cdot\left(\sum_{i=1}^{+\infty} \frac{\left|\nabla X_{i}(x)\right|^{2}}{\lambda_{i}^{2}}\right)^{1 / 2} \\
& +\int_{0}^{t} \frac{1}{\psi(\tau, \alpha)}\left(\sum_{i=1}^{+\infty} \lambda_{i}^{2}\left(F, X_{i}\right)^{2}\right)^{1 / 2} \\
& \cdot\left(\sum_{i=1}^{+\infty} \frac{\left|\nabla X_{i}(x)\right|^{2}}{\lambda_{i}^{2}}\right)^{1 / 2} d \tau \\
& \leq\left(\left\|A\left(u_{0}\right)\right\|_{2}+C\|A(F)\|_{2}\right)\left(\sum_{i=1}^{+\infty} \frac{\left|\nabla X_{i}(x)\right|^{2}}{\lambda_{i}^{2}}\right)^{1 / 2} \text {, }
\end{aligned}
$$

where $C=\int_{0}^{T}(1 / \psi(\tau, \alpha)) d \tau$. Consequently, the series (66) uniformly converges to $\nabla u$ and the generalized solution (54) belongs to $C_{x}^{1}(D)$.

Applying the similarly method, we can show the generalized solution (54) belongs to $C_{x}^{2}(D)$ as well and the relation

$$
\begin{aligned}
\Delta u= & \sum_{i=1}^{+\infty}\left(u_{0}, X_{i}\right) e^{-\int_{0}^{t} \frac{\lambda_{i}}{\psi(\tau, \alpha)} d \tau} \Delta X_{i}(x) \\
& +\sum_{i=1}^{+\infty} e^{-\int_{0}^{t} \frac{\lambda_{i}}{\psi(\tau, \alpha)} d \tau} \int_{0}^{t} e^{\int_{0}^{\tau} \frac{\lambda_{i}}{\psi(s, \alpha)} d s} \frac{\left(F(x, \tau), X_{i}(x)\right) \Delta X_{i}(x)}{\psi(\tau, \alpha)} d \tau
\end{aligned}
$$

holds true. In fact, since $A\left(u_{0}\right) \in M_{A}, A(F) \in M_{A}$, then $A^{2}\left(u_{0}\right) \in L^{2}(D), A^{2}(F) \in L^{2}(D)$. Moreover, due to $A^{2}\left(u_{0}\right)$, $A^{2}(F) \in M_{A}$, so $A^{2}\left(u_{0}\right)$ and $A^{2}(F)$ can be expanded into uniformly convergent series for any $t \in(0, T)$. Note that the series $\sum_{i=1}^{+\infty}\left(\left|\Delta X_{i}\right|^{2} / \lambda_{i}^{3}\right)$ is uniformly convergent (see [38]), then by the Cauchy inequality, we have

$$
\begin{aligned}
\sum_{i=1}^{+\infty} & \left|\left(F(x, t), X_{i}(x)\right) \Delta X_{i}(x)\right| \\
\leq & \sum_{i=1}^{+\infty}\left|\lambda_{i}^{\frac{3}{2}}\left(F(x, t), X_{i}(x)\right)\right| \cdot\left|\frac{\Delta X_{i}(x)}{\lambda_{i}^{3 / 2}}\right| \\
\leq & l_{1}\left(\sum_{i=1}^{+\infty} \lambda_{i}^{4}\left|\left(F(x, t), X_{i}(x)\right)\right|^{2}\right)^{1 / 2} \\
& \left.\cdot\left(\sum_{i=1}^{+\infty}\left(\frac{\left|\Delta X_{i}(x)\right|^{2}}{\lambda_{i}^{3}}\right)\right)^{1 / 2}\right) \\
\leq & l_{1}\left\|A^{2}(F)\right\|_{2}\left(\sum_{i=1}^{+\infty}\left(\frac{\left|\Delta X_{i}(x)\right|^{2}}{\lambda_{i}^{3}}\right)\right)^{1 / 2} .
\end{aligned}
$$

Due to $\lim _{i \rightarrow+\infty} \lambda_{i}=+\infty$, there exists a constant $l$ such that $\lambda_{i}^{3}<l \lambda_{i}^{4}, i=1,2, \cdots$, where $l_{1}$ is a constant depending on $l$. Then (73) implies that the series $\sum_{i=1}^{+\infty} \mid(F(x, t)$, $\left.X_{i}(x)\right) \Delta X_{i}(x) \mid$ is uniformly convergent on $D$ for any $t \in$ $(0, T)$. Using the above facts and applying the Parseval equality and Cauchy inequality, we get

$$
\begin{aligned}
& \sum_{i=1}^{+\infty}\left|\left(u_{0}, X_{i}\right) e^{-\int_{0}^{t} \frac{\lambda_{i}}{\psi(\tau, \alpha)} d \tau} \Delta X_{i}(x)\right| \\
& +\sum_{i=1}^{+\infty}\left|e^{-\int_{0}^{t} \frac{\lambda_{i}}{\psi(\tau, \alpha)} d \tau} \int_{0}^{t} e^{\int_{0}^{\tau} \frac{\lambda_{i}}{\psi(s, \alpha)} d s} \frac{\left(F(x, \tau), X_{i}(x)\right) \Delta X_{i}(x)}{\psi(\tau, \alpha)} d \tau\right| \\
& \leq \sum_{i=1}^{+\infty}\left|\left(u_{0}, X_{i}\right) \Delta X_{i}(x)\right|+\sum_{i=1}^{+\infty}\left|\int_{0}^{t} \frac{\left(F(x, \tau), X_{i}(x)\right) \Delta X_{i}(x)}{\psi(\tau, \alpha)} d \tau\right| \\
& \leq \sum_{i=1}^{+\infty}\left|\lambda_{i}^{\frac{3}{2}}\left(u_{0}, X_{i}\right)\right| \cdot\left|\frac{\Delta X_{i}(x)}{\lambda_{i}^{3 / 2}}\right| \\
& +\int_{0}^{t} \frac{1}{\psi(\tau, \alpha)}\left(\sum_{i=1}^{+\infty}\left|\lambda_{i}^{\frac{3}{2}}\left(F(x, \tau), X_{i}(x)\right)\right| \cdot\left|\frac{\Delta X_{i}(x)}{\lambda_{i}^{3 / 2}}\right|\right) d \tau \\
& \leq l_{1}\left(\sum_{i=1}^{+\infty} \lambda_{i}^{4}\left(u_{0}, X_{i}\right)^{2}\right)^{1 / 2} \cdot\left(\sum_{i=1}^{+\infty} \frac{\left|\Delta X_{i}(x)\right|^{2}}{\lambda_{i}^{3}}\right)^{1 / 2} \\
& +\int_{0}^{t} \frac{l_{1}}{\psi(\tau, \alpha)}\left(\sum_{i=1}^{+\infty} \lambda_{i}^{4}\left(F, X_{i}\right)^{2}\right)^{1 / 2} \\
& \left(\sum_{i=1}^{+\infty} \frac{\left|\Delta X_{i}(x)\right|^{2}}{\lambda_{i}^{3}}\right)^{1 / 2} d \tau \\
& \leq\left(l_{1}\left\|A^{2}\left(u_{0}\right)\right\|_{2}+C l_{1}\left\|A^{2}(F)\right\|_{2}\right)\left(\sum_{i=1}^{+\infty} \frac{\left|\Delta X_{i}(x)\right|^{2}}{\lambda_{i}^{3}}\right)^{1 / 2} .
\end{aligned}
$$

Consequently, the relation (72) holds true and the generalized solution (54) belongs to $C_{x}^{2}(D)$. It remains to prove that (54) belongs to $C_{t}^{1}(0, T)$. We take a derivative term by term with respect to $t$ (54) and construct the series 


$$
\begin{aligned}
& -\sum_{i=1}^{+\infty} \frac{\lambda_{i}\left(u_{0}, X_{i}\right)}{\psi(t, \alpha)} e^{-\int_{0}^{t} \frac{\lambda_{i}}{\psi(t, \alpha)} d \tau} X_{i}(x) \\
& -\sum_{i=1}^{+\infty} \frac{\lambda_{i} e^{-\int_{0}^{t}\left(\lambda_{i} / \psi(\tau, \alpha)\right) d \tau}}{\psi(t, \alpha)} \int_{0}^{t} e \int_{0}^{\tau} \frac{\lambda_{i}}{\psi(s, \alpha)} d s \frac{\left(F(x, \tau), X_{i}(x)\right) X_{i}(x)}{\psi(\tau, \alpha)} d \tau \\
& +\sum_{i=1}^{+\infty} \frac{\left(F(x, \tau), X_{i}(x)\right) X_{i}(x)}{\psi(t, \alpha)} .
\end{aligned}
$$

Now let $t \in[\varepsilon, T], 0<\varepsilon<T$. Note that $1 / \psi(t, \alpha)$ is the Riemann integrable on $[\varepsilon, T]$; there exists $M>0$ such that $|1 / \psi(t, \alpha)| \leq M$. Next we shall to prove that the above series (75) is uniformly convergent on any interval $[\varepsilon, T]$, $0<\varepsilon<T$. By the uniformly convergence of $\sum_{i=1}^{+\infty}\left(\left|X_{i}\right|^{2} / \lambda_{i}\right)$ (see [38]), applying the Parseval equality and the Cauchy inequality, we get

$$
\begin{aligned}
\sum_{i=1}^{+\infty} \mid & \frac{\lambda_{i}\left(u_{0}, X_{i}\right)}{\psi(t, \alpha)} e^{-\int_{0}^{t} \frac{\lambda_{i}}{\psi(\tau, \alpha)} d \tau} X_{i}(x)\left|+\sum_{i=1}^{+\infty}\right| \frac{\left(F(x, \tau), X_{i}(x)\right) X_{i}(x)}{\psi(t, \alpha)} \mid \\
& +\sum_{i=1}^{+\infty}\left|\frac{\lambda_{i} e^{-\int_{0}^{t}\left(\lambda_{i} / \psi(\tau, \alpha)\right) d \tau}}{\psi(t, \alpha)} \int_{0}^{t} e^{\int_{0}^{\tau} \frac{\lambda_{i}}{\psi(s, \alpha)} d s} \frac{\left(F(x, \tau), X_{i}(x)\right) X_{i}(x)}{\psi(\tau, \alpha)} d \tau\right| \\
\leq & \sum_{i=1}^{+\infty} M\left|\lambda_{i}\left(u_{0}, X_{i}\right) X_{i}(x)\right|+\sum_{i=1}^{+\infty} M\left|\left(F(x, \tau), X_{i}(x)\right) X_{i}(x)\right| \\
& +\sum_{i=1}^{+\infty} \int_{0}^{t} \frac{M \lambda_{i}\left|\left(F(x, \tau), X_{i}(x)\right) X_{i}(x)\right|}{\psi(\tau, \alpha)} d \tau \\
\leq & \sum_{i=1}^{+\infty} M\left|\lambda_{i}^{3 / 2}\left(u_{0}, X_{i}\right)\right| \cdot\left|\frac{X_{i}(x)}{\lambda_{i}^{1 / 2}}\right|+\sum_{i=1}^{+\infty} M\left|\lambda_{i}^{1 / 2}\left(F, X_{i}\right)\right| \cdot\left|\frac{X_{i}(x)}{\lambda_{i}^{1 / 2}}\right| \\
& +\int_{0}^{t} \frac{M}{\psi(\tau, \alpha)}\left(\sum_{i=1}^{+\infty}\left|\lambda_{i}^{3 / 2}\left(F(x, \tau), X_{i}(x)\right)\right| \cdot \mid \frac{X_{i}(x)}{\left.\lambda_{i}^{1 / 2} \mid\right) d \tau}\right. \\
\leq & M_{1}\left(\sum_{i=1}^{+\infty} \lambda_{i}^{4}\left(u_{0}, X_{i}\right)^{2}\right)^{1 / 2} \cdot\left(\sum_{i=1}^{+\infty} \frac{\left|X_{i}(x)\right|^{2}}{\lambda_{i}}\right)^{1 / 2} \\
& +M_{2}\left(\sum_{i=1}^{+\infty} \lambda_{i}^{2}\left(F, X_{i}\right)^{2}\right)^{1 / 2} \cdot\left(\sum_{i=1}^{+\infty} \frac{\left|X_{i}(x)\right|^{2}}{\lambda_{i}}\right)^{1 / 2} \\
& +\int_{0}^{t} \frac{M_{1}}{\psi(\tau, \alpha)}\left(\sum_{i=1}^{+\infty} \lambda_{i}^{4}\left(F(x, \tau), X_{i}\right)^{2}\right)^{1 / 2} \\
& \cdot\left(\sum_{i=1}^{+\infty} \frac{\left|X_{i}(x)\right|^{2}}{\lambda_{i}}\right)^{1 / 2} d \tau \\
\leq & \left(M_{1}|| A^{2}\left(u_{0}\right)\left\|_{2}+M_{2}\right\| A(F)\left\|_{2}+C M_{1}\right\| A^{2}(F) \|_{2}\right) \\
& \cdot\left(\sum_{i=1}^{+\infty} \frac{\left|X_{i}(x)\right|^{2}}{\lambda_{i}}\right)^{1 / 2} \cdot
\end{aligned}
$$

Once again, due to $\lim _{i \rightarrow+\infty} \lambda_{i}=+\infty$, there exists a constant $l$ such that $\lambda_{i}^{3}<l \lambda_{i}^{4}, \lambda_{i}<l \lambda_{i}^{2} i=1,2, \cdots$, where $M_{1}$, $M_{2}$ are some constants depending on $l$ and $M$. Then, the series (75) uniformly convergent on any interval $[\varepsilon, T]$,
$0<\varepsilon<T$. Therefore, the generalized solution (54) belongs to $C_{t}^{1}(0, T)$ and the proof is complete.

Theorem 19. The classical solution (50) of (1) and (36) with $F(x, t) \equiv 0$ decay to zero with the rate $e^{-\int_{0}^{t}\left(\lambda_{1} / \psi(s, \alpha)\right) d s}$.

Proof. In fact, since $u_{0} \in M_{A}$, then $\left|\sum_{i=1}^{+\infty}\left(u_{0}, X_{i}\right) X_{i}(x)\right|=\left|u_{0}\right|$ $<+\infty$. Then for any $t>0$ and $x \in \bar{D}$, we have

$$
\begin{aligned}
& \left|\sum_{i=1}^{+\infty}\left(u_{0}, X_{i}\right) e^{-\int_{0}^{t} \frac{\lambda_{i}}{\psi(\tau, \alpha)} d \tau} X_{i}(x)\right| \\
& \leq e^{-\int_{0}^{t} \frac{\lambda_{1}}{\psi(s, \alpha)} d s}\left|\sum_{i=1}^{+\infty}\left(u_{0}, X_{i}\right) e^{-\int_{0}^{t} \frac{\lambda_{i}-\lambda_{1}}{\psi(\tau, \alpha)} d \tau} X_{i}(x)\right| \\
& \leq\left|u_{0}\right| e^{-\int_{0}^{t} \frac{\lambda_{1}}{\psi(s, \alpha)} d s} \longrightarrow 0(\text { ast } \longrightarrow+\infty) .
\end{aligned}
$$

\section{Conclusions}

The GCFD is an extension of the classical derivatives and the conformable derivatives. In this paper, we introduce its fractional Taylor power series expansions and prove the theorem of term-by-term integration and differentiation. Utilizing its properties, we obtain the uniqueness and existence of the classical solution of the classical diffusion equations with GCFD by using variable separation method.

\section{Data Availability}

No data were used to support this study.

\section{Conflicts of Interest}

The authors declare that they have no competing interests.

\section{Authors' Contributions}

The authors contributed equally and significantly in writing this paper. All authors read and approved the final manuscript.

\section{Acknowledgments}

This work was supported by the National Natural Science Foundation of China (No. 11671181). The author is very grateful to all editors and referees for their valuable suggestions.

\section{References}

[1] K. Diethelm, The analysis of fractional differential equations, Lecture Notes in Mathematics, Berlin, 2010.

[2] A. A. Kilbas, H. M. Struvasteva, and J. J. Trujilio, Theory and applications of fractional differential equations, North-Holland Mathematics Studies, Amsterdam, 2006.

[3] Y. Zhou, Basic theory of fractional differential equations, World Scientific, London, 2014.

[4] Y. Zhou, Fractional evolution equations and inclusions: analisis and control, Elsevier Science and Technology, London, 2016. 
[5] R. Metzler and J. Klafter, "The random walk's guide to anomalous diffusion: a fractional dynamics approach," Physics Reports, vol. 339, no. 1, pp. 1-77, 2000.

[6] X. Zhang, L. Liu, and Y. Wu, "Multiple positive solutions of a singular fractional differential equation with negatively perturbed term," Mathematical and Computer Modelling, vol. 55, no. 3-4, pp. 1263-1274, 2012.

[7] X. Zhang, Y. Wu, and L. Caccetta, "Nonlocal fractional order differential equations with changing-sign singular perturbation," Applied Mathematical Modelling, vol. 39, no. 21, pp. 6543-6552, 2015.

[8] Y. Cui, W. Ma, Q. Sun, and X. Su, "New uniqueness results for boundary value problem of fractional differential equation," Nonlinear Analysis, vol. 23, pp. 31-39, 2018.

[9] C. Chen, K. Li, Y. Chen, and Y. Huang, "Two-grid finite element methods combined with Crank-Nicolson scheme for nonlinear Sobolev equations," Advances in Computational Mathematics, vol. 45, no. 2, pp. 611-630, 2019.

[10] C. Chen, X. Zhang, G. Zhang, and Y. Zhang, "A two-grid finite element method for nonlinear parabolic integro-differential equations," International Journal of Computer Mathematics, vol. 96, no. 10, pp. 2010-2023, 2018.

[11] C. Chen, W. Liu, and C. Bi, "A two-grid characteristic finite volume element method for semilinear advection-dominated diffusion equations," Numerical Methods for Partial Differential Equations, vol. 29, no. 5, pp. 1543-1562, 2013.

[12] C. Chen and X. Zhao, "A posteriori error estimate for finite volume element method of the parabolic equations," Numerical Methods for Partial Differential Equations, vol. 33, no. 1, pp. 259-275, 2017.

[13] C. Bi, Y. Lin, and M. Yang, "Finite volume element method for monotone nonlinear elliptic problems," Numerical Methods for Partial Differential Equations, vol. 29, no. 4, pp. 10971120, 2013.

[14] C. Bi and M. Liu, "A discontinuous finite volume element method for second order elliptic problems," Numerical Methods for Partial Differential Equations, vol. 28, no. 2, pp. 425-440, 2012.

[15] M. Yang, "Higher-order finite volume element methods based on Barlow points for one-dimensional elliptic and parabolic problems," Numerical Methods for Partial Differential Equations, vol. 31, no. 4, pp. 977-994, 2015.

[16] X. Zhang, J. Xu, J. Jiang, Y. Wu, and Y. Cui, “The convergence analysis and uniqueness of blow-up solutions for a dirichlet problem of the general k-hessian equations," Applied Mathematics Letters, vol. 102, article 106124, 2020.

[17] X. Zhang, J. Jiang, Y. Wu, and Y. Cui, “The existence and nonexistence of entire large solutions for a quasilinear Schrödinger elliptic system by dual approach," Applied Mathematics Letters, vol. 100, article 106018, 2020.

[18] X. Wang and Q. Fan, “A modified Ren's method with memory using a simple self-accelerating parameter," Mathematics, vol. 8 , no. 4 , p. $540,2020$.

[19] X. Wang and Y. Tao, "A new newton method with memory for solving nonlinear equations," Mathematics, vol. 8, no. 1, p. 108, 2020.

[20] R. Khalil, M. Al Horani, A. Yousef, and M. Sababheh, "A new definition of fractional derivative," Journal of Computational and Applied Mathematics, vol. 264, pp. 65-70, 2014.

[21] T. Abdeljawad, "On conformable fractional calculus," Journal of Computational and Applied Mathematics, vol. 279, pp. 57-66, 2015.
[22] D. Zhao and M. Luo, "General conformable fractional derivative and its physical interpretation," Calcolo, vol. 54, no. 3, pp. 903-917, 2017.

[23] D. Zhao, X. Pan, and M. Luo, "A new framework for multivariate general conformable fractional calculus and potential applications," Physica A: Statistical Mechanics and its Applications, vol. 510, pp. 271-280, 2018.

[24] S. Zhang, S. Li, and L. Hu, "The existeness and uniqueness result of solutions to initial value problems of nonlinear diffusion equations involving with the conformable variable derivative," Revista de la Real Academia de Ciencias Exactas, Físicas y Naturales. Serie A. Matemáticas, vol. 113, pp. 1601-1623, 2019.

[25] Z. Bai, Y. Cheng, and S. Sun, "On solutions of a class of threepoint fractional boundary value problems," Boundary Value Problems, vol. 2020, no. 1, 2020.

[26] S. Yang, L. Wang, and S. Zhang, "Conformable derivative: Application to non-Darcian flow in low-permeability porous media," Applied Mathematics Letters, vol. 79, pp. 105-110, 2018.

[27] H. W. Zhou, S. Yang, and S. Q. Zhang, "Conformable derivative approach to anomalous diffusion," Physica A: Statistical Mechanics and its Applications, vol. 491, pp. 1001-1013, 2018.

[28] D. Avci, E. İskender Beyza, and N. Özdemir, "The dirichlet problem of a conformable advection-diffusion equation," Thermal Science, vol. 21, 1, Part A, pp. 9-18, 2017.

[29] W. S. Chung, "Fractional newton mechanics with conformable fractional derivative," Journal of Computational and Applied Mathematics, vol. 290, pp. 150-158, 2015.

[30] Y. Luchko, "Maximum principle for the generalized timefractional diffusion equation," Journal of Mathematical Analysis and Applications, vol. 351, no. 1, pp. 218-223, 2009.

[31] Y. Luchko, "Some uniqueness and existence results for the initial-boundary-value problems for the generalized timefractional diffusion equation," Computers \& Mathematcs with Applications, vol. 59, no. 5, pp. 1766-1772, 2010.

[32] M. Al-Refai and Y. Luchko, "Maximum principle for the fractional diffusion equations with the Riemann-Liouville fractional derivative and its applications," Fractional Calculus and Applied Analysis, vol. 17, no. 2, pp. 483-498, 2014.

[33] M. Al-Refai and Y. Luchko, "Maximum principle for the multi-term time-fractional diffusion equations with the Riemann-Liouville fractional derivatives," Applied Mathematics and Computation, vol. 257, pp. 40-51, 2015.

[34] M. Borikhanov, M. Kirane, and B. T. Torebek, "Maximum principle and its application for the nonlinear time-fractional diffusion equations with Cauchy-Dirichlet conditions," Applied Mathematics Letters, vol. 81, pp. 14-20, 2018.

[35] C. Chen, H. Liu, X. Zheng, and H. Wang, "A two-grid MMOC finite element method for nonlinear variable-order timefractional mobile/immobile advection-diffusion equations," Computers \& Mathematcs with Applications, vol. 79, no. 9, pp. 2771-2783, 2020.

[36] Q. Zhang, H.-R. Sun, and Y. Li, "Global existence and blow-up of solutions of the Cauchy problem for a time fractional diffusion system," Computers \& Mathematics with Applications, vol. 78, no. 5, pp. 1357-1366, 2019.

[37] V. S. Vladimiov, Equations of mathematical physics, Steklov Institute of Mathematics, Moscow, 1971.

[38] Y. Luchko, "Initial-boundary-value problems for the onedimensional time-fractional diffusion equation," Fractional Calculus and Applied Analysis, vol. 15, no. 1, pp. 141-160, 2012. 